\title{
A time-series study of the health effects of water-soluble and total- extractable metal content of airborne particulate matter
}

\author{
Mathew R. Heal, ${ }^{1}$ Robert A. Elton, ${ }^{2}$ Leon R. Hibbs, ${ }^{1, a}$ Raymond M. Agius, ${ }^{3}$ and Iain J. \\ Beverland, ${ }^{4}$
}

${ }^{1}$ School of Chemistry, University of Edinburgh, Edinburgh, UK.

${ }^{2}$ Division of Community Health Sciences, University of Edinburgh, Edinburgh, UK.

${ }^{3}$ Centre for Occupational and Environmental Health, University of Manchester, Manchester, UK.

${ }^{4}$ Department of Civil Engineering, University of Strathclyde, Glasgow, UK.

${ }^{a}$ Present address: Reigate and Banstead Borough Council, Town Hall, Castlefield Road, Reigate, Surrey RH2 OSH, UK

\section{Corresponding author:}

Dr. Mathew R. Heal, School of Chemistry, University of Edinburgh, West Mains Road, Edinburgh

EH9 3JJ

Email:m.heal@ed.ac.uk tel: 01316504764 fax: 01316506453

Key words:

Time series, epidemiology, $\mathrm{PM}_{10}$, transition metal, cardiovascular 


\section{WHAT THIS PAPER ADDS}

- Many time-series studies have demonstrated significant positive associations between mass concentration of ambient particulate matter and adverse acute cardiopulmonary health outcomes.

- However, the hypothesis, based principally on toxicological evidence, that the concentrations of some metals in PM are explanatory factors for the health outcomes, has not been tested directly in the epidemiological context.

- Within the limitations of the study power, analysis of 1 year of direct chemical determination of daily particle-bound metals, and of a further 7 years of reconstructed estimated daily metals exposures, showed no evidence of significant associations between particle-bound metal concentrations and cardiopulmonary health outcomes independent of PM mass.

- Metal content may still be a causal agent in adverse responses from exposure to PM, but the generally strong correlations between metal and total PM observed here suggests that unambiguous quantification of associations between adverse health outcomes and PM metal exposure will be difficult, even using more powerful time series of direct measurements. 


\section{ABSTRACT}

Objectives: To assess whether adverse acute cardiopulmonary health outcomes are associated with concentration of trace metals in airborne particulate matter.

Methods: Daily $\mathrm{PM}_{10}$ and $\mathrm{PM}_{2.5}$ was collected for one year in Edinburgh, UK, and the watersoluble and total-extractable content of 11 trace metals determined in each sample. Time series were analysed using generalised additive Poisson regression models, including adjustment for minimum temperature and loess smoothing of trends. Methods were explored of extending the time series of metal concentration in daily $\mathrm{PM}_{10}$ for the previous 7 years using multiple regression of the variation in metal content for the 1 year of measurements and the associated variation in air mass source region and other concurrently-measured potential predictor variables.

Results: The 1 year of direct measurements showed no evidence of significant associations of particle-bound metal concentration with health outcomes beyond that expected by chance. Analysis of the extended time series showed significant positive associations with cardiovascular admissions both for total $\mathrm{PM}_{10}$ and for a number of the metals (e.g. $\mathrm{Cu}, \mathrm{Fe}, \mathrm{Ni}$, $\mathrm{V}, \mathrm{Zn}$ ) but the metal effects were no longer significant after adjusting for $\mathrm{PM}_{10}$.

Conclusions: Within the limitations of its power, the epidemiological results have not provided evidence for associations between particle-bound metal concentrations and adverse health outcomes that are substantially greater than for total PM. The generally strong correlations between metal and total PM suggest that quantifying independent effects of PM metal exposure on health will be difficult, even using more powerful time series of direct measurements.

\section{INTRODUCTION}

Many time-series studies have demonstrated significant positive associations between mass concentration of ambient particulate matter (PM) and acute impacts on human morbidity and mortality. ${ }^{1}$ However, PM is highly diverse in size and chemical composition, so an important question is whether specific chemical properties are responsible for the adverse health associations observed with PM mass, which may then allow better-targetted abatement strategies. There has been some progress towards answering the question, predominantly through toxicological rather than epidemiological studies, but considerable uncertainty remains. $^{2}$

A number of toxicological studies ${ }^{3-5}$ have shown that transition metal content of PM (especially $\mathrm{Fe}, \mathrm{Cu}, \mathrm{V}, \mathrm{Zn}$ ) promotes in vitro and in vivo inflammation response, mediated by reactive oxygen species generated through redox reactions of the metals. ${ }^{6}$ However, the hypothesis, based on the toxicological evidence, that the concentrations of some metals in ambient PM are explanatory factors for adverse acute cardiopulmonary health outcomes, has not been tested directly in the epidemiological context because of lack of sufficient chemically-speciated ambient measurements. This work is the first direct study of the quantitative relationship between respiratory and cardiovascular mortality and morbidity and daily concentration of metals in $\mathrm{PM}_{10}$ and $\mathrm{PM}_{2.5}$ size fractions.

\section{METHODS}

\section{Metal exposure data}

Simultaneous samples of $\mathrm{PM}_{10}$ and $\mathrm{PM}_{2.5}$ were collected daily for 1 year (1999-2000) at an urban background site in central Edinburgh, UK (population 450,000) using Partisol 2025 
units (R \& P, USA). ${ }^{7}$ Each filter was extracted sequentially with $18 \mathrm{M} \Omega$ ultrapure water at 37 ${ }^{\circ} \mathrm{C}$ (the "water-soluble" fraction), followed by a 2.8:1 v/v mixture of concentrated $\mathrm{HCl}$ and $\mathrm{HNO}_{3}$ acids at $190{ }^{\circ} \mathrm{C}$, and the concentration of $\mathrm{As}, \mathrm{Cd}, \mathrm{Cr}, \mathrm{Cu}, \mathrm{Fe}, \mathrm{Mn}, \mathrm{Ni}, \mathrm{Pb}, \mathrm{Ti}, \mathrm{V}$ and $\mathrm{Zn}$ in each extract quantified using ICP-MS (VG Elemental PasmaQuad). ${ }^{8}$ The sum of the metal yield from both extractions gave the "total-extractable" metal content of PM for that sample. Metal data are expressed as both $\mathrm{ng} \mathrm{m}^{-3}$ airborne concentration and $\mathrm{ng} \mathrm{g}^{-1}$ metal:particle mass factor. Full detail of procedures, and of the concentrations measured, are given elsewhere. ${ }^{7,8}$

\section{Epidemiological methods}

Data for deaths and hospital admissions in City of Edinburgh post-code sectors were obtained from the Information and Statistics Division of National Health Service Scotland. Five different health outcomes were considered (numbers of hospital admissions and deaths from respiratory and cardiovascular causes plus deaths from all causes), separated into two age groups (65 and over, and under 65).

The numbers of deaths and admissions were tested for association with particle-bound metal concentration by generalised additive Poisson regression (GAM) models using S-PLUS software. This method involves the use of loess (locally weighted regression) to fit smooth curves to the patterns of incidence over time, with the degree of smoothness determined by the width of the time window used in the regression. Confidence limits for effect sizes can be derived by examining the difference in deviance when the pollution term is included or excluded from a fit involving the other terms chosen.

GAMs with 30-day windows were applied to each combination of metal, PM fraction, health outcome and age group, using the average of the previous three days' particle-bound metal concentrations and also adjusting for day of the week and minimum temperature, with the latter also fitted by loess but with a relatively coarse smoothing. A loess fit using a 30-day smoothing window dealt sensibly with the over-dispersion in some health outcome data arising from seasonal and short-term variations in number of events (e.g. an influenza epidemic). Sensitivity analyses with different loess window sizes or pollution lags did not change the general conclusions.

With 11 different metals measured in four different PM fractions being correlated with five different health outcomes in two different age groups, a total of 440 significance test results are available, and thus the issue of multiple testing clearly arises. Correspondingly, a subset of 60 "primary" analyses was defined using water-soluble and total particle-bound concentrations of five metals $(\mathrm{Cu}, \mathrm{Fe}, \mathrm{Ni}, \mathrm{V}$ and $\mathrm{Zn})$ that were the best a priori candidates for having adverse effects on health, and also on three health outcomes based on previous results for Edinburgh ${ }^{9}$ (cardiovascular admissions and respiratory and all-cause deaths in those aged over 65). The remaining "secondary" analyses were screened for more than the expected number of formally significant results.

\section{Estimation of additional metal exposure}

Multivariate regression was explored as a way of estimating the metal content of daily $\mathrm{PM}_{10}$ for the 7 years prior to the one year of direct measurements. The regression variables tested were the 30 variables of distance north and distance east of Edinburgh, and pressure level, for the air mass back trajectories at $12,24, \ldots 120$ hours prior to daily arrivals in Edinburgh (calculated using the trajectory model at the British Atmospheric Data Centre (www.badc.rl.ac.uk)), plus daily mean windspeed, daily rainfall, minimum and maximum temperature, and daily mean concentrations of the co-pollutants $\mathrm{NO}_{2}, \mathrm{CO}, \mathrm{SO}_{2}$ and $\mathrm{O}_{3}$. The 
approach was predicated on the observation that air-mass source region (i.e. long-range transport) contributes to variation in PM chemical composition. ${ }^{8}$ The regression equations of significant predictor variables for metal:particle mass factors derived from the one year of measurements were applied to archived daily PM $_{10}$ between 1992 and 1999.

\section{RESULTS}

Confidence limits for the effects associated with the measured metal data for the 60 "primary" analyses are shown in Table 1. Only four of the 60 analyses are significant (which is no more than would be expected if no true associations), and in each case the estimate suggests a decreasing risk with increasing exposure to particle-bound metal.

Table 1: Approximate 95\% confidence limits for percent changes in relative risk in subjects aged $\geq 65$ for three health outcomes over the interquartile range from one year of daily measurements of particle-bound metal concentrations (unadjusted for $\mathrm{PM}_{10}$ or $\mathrm{PM}_{2.5}$ mass). The relative risks associated with $\mathrm{PM}_{10}$ and $\mathrm{PM}_{2.5}$ mass are given in the bottom two rows. Significant results are shown as $*=P<0.05$ or $* *=P<0.01$. The $R^{2}$ values in the penultimate column indicate the power of the regression models to predict the one year of measured particle-bound metal concentrations. The final column is the square of the Spearman rank correlation coefficient between daily metal concentration in the $\mathrm{PM}_{2.5}$ or $\mathrm{PM}_{10}$ fractions and either total $\mathrm{PM}_{2.5}$ or $\mathrm{PM}_{10}$ for the year of measurements and provides an indication of the strength of the predictive relationship between total PM and its metal content.

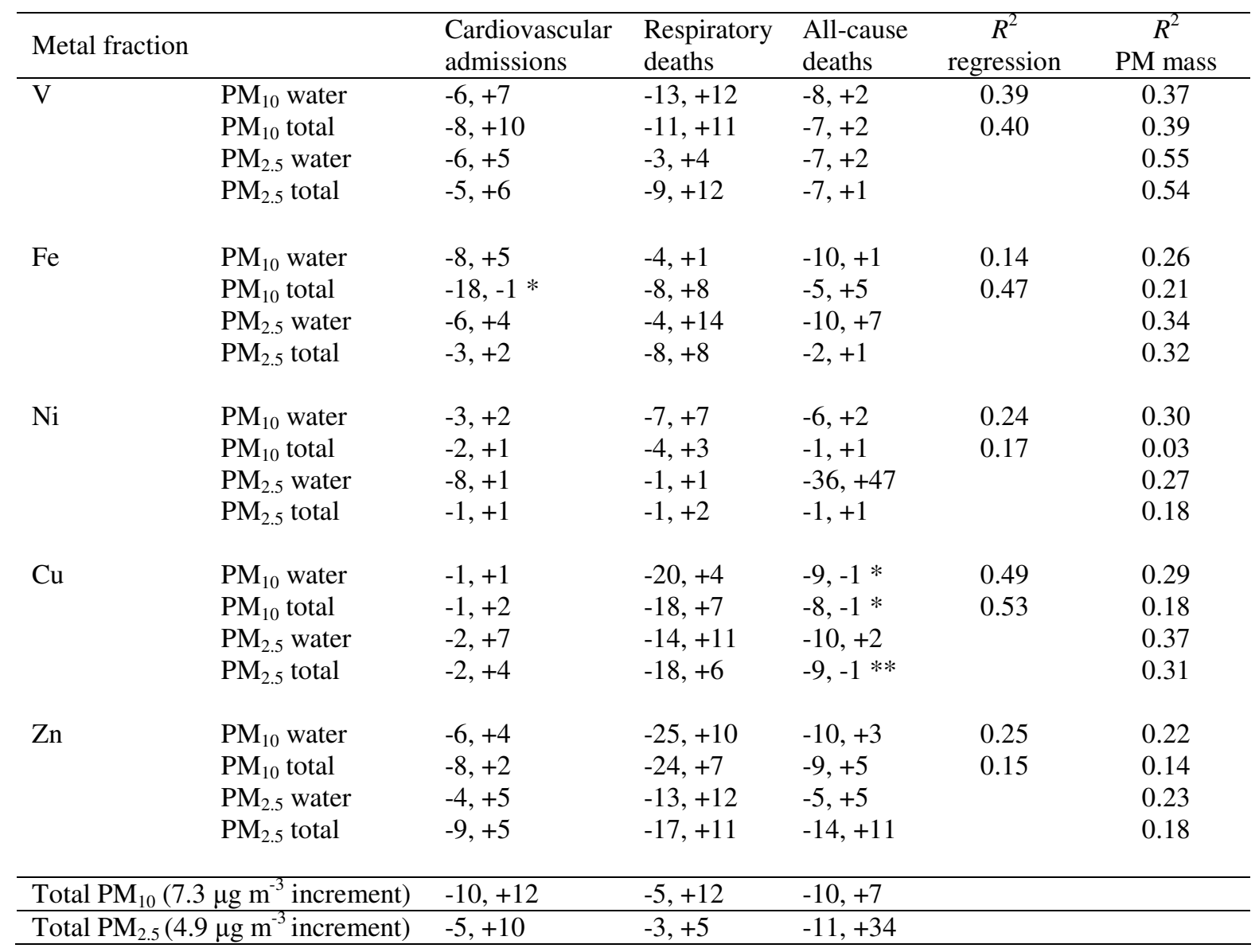


Only three of the other 172 "secondary" analyses in those aged over 65 showed significant health associations with metal concentrations, which is less than expected by chance. Of the 220 analyses in younger subjects, six at $P<0.05$ showed no particular pattern, but a further nine at $P<0.05$ and eight at $P<0.01$ were all for cardiovascular admissions. Again the coefficients were negative, so no suggestion of high risk from the metals concerned.

For the extended time series there was a significant association with cardiovascular admissions for total $\mathrm{PM}_{10}$ concentration (95\% CI of $\mathrm{RR}+1,+6$, for $10 \mu \mathrm{g} \mathrm{m}^{-3}$ increment), consistent with previous findings for Edinburgh ${ }^{9}$, and also for a number of the metals, both water soluble and total extractable fractions. However, further multiple regressions showed that the metal effects were no longer significant after adjusting for total $\mathbf{P M}_{10}$ (data not presented).

\section{DISCUSSION}

The results of this study are essentially negative, in that no more significant results for association of health effects with particle-bound metal concentrations are observed than expected than by chance alone, whether from the one year of measurements or from the attempt at additional metal exposure estimation. However, the extent to which this is a "positively negative" conclusion clearly depends on the statistical power of the study, and even a year-long effort to collect measurement data is a relatively short period. For the measured data, the confidence intervals in Table 1 are of the order of 5-20\% and need to be interpreted in the context of other much larger studies demonstrating effect sizes of the order of $1 \%$ on relative risk over corresponding ranges of total PM. ${ }^{1}$ It is therefore reasonable to state that it is unlikely that the effects on health associated with any of the particle-bound metal metrics are more than around 5 to 20 times as great as those of the total PM. The similar pattern of results for the eight-year extrapolation of metal concentrations to those for total $\mathrm{PM}_{10}$ is perhaps not surprising given that particle-bound metal concentrations and total PM are fairly strongly correlated with one another, as shown by the squared correlation coefficients in the final column of Table 1.

This work does not necessarily show that no such health associations exist, but that the methodology used here has not detected them if they do. There are two aspects to this. First, as mentioned above, for most metals a large proportion of the day-to-day variation in metal concentration arises from variation in total $\mathrm{PM}_{10}$ rather than from the proportion of $\mathrm{PM}_{10}$ composed of that metal. Secondly, the attempt to estimate daily particle-bound metal concentrations through regression of other data was not particularly effective as illustrated by the $R^{2}$ values in the penultimate column of Table 1. Examination of confidence limits for the metal:particle mass factors in a multiple regression analysis of health outcomes adjusting for total $\mathrm{PM}_{10}$ suggest that the gain in power from using the longer time series was almost entirely offset by the loss from the extrapolation process.

One pan-European time-series study also failed to find conclusive effects from $\mathrm{Fe}$ in $\mathrm{PM}_{10}{ }^{10}$ A few studies have investigated associations between adverse health outcome and putative sources of PM assigned statistically using metals as chemical tracers, ${ }^{11,12}$ but none of these studies were capable of isolating the potential health effect of individual metals.

The difficulty of separately quantifying the effects of metals in real samples of PM, from that of total PM with which they correlate quite strongly, was also encountered in a toxicological study using daily samples of $\mathrm{PM}_{10}$ taken from six UK sites. ${ }^{13}$ Overall, the markers of in vivo 
and in vitro inflammation examined were most strongly influenced by total PM mass dose. The independent effect on some of the toxicological end points of certain trace metals within the PM (e.g. water-soluble $\mathrm{Zn}$ and $\mathrm{Mn}$ ) were less strong.

In conclusion, this study, albeit relatively low powered, has not provided evidence for associations between particle-bound metal concentrations and adverse health outcomes that are substantially greater than for total PM. The generally strong correlations observed between metal and total PM concentrations in urban air suggest that distinguishing independent effects will be difficult, even using more powerful time series of direct measurements.

\section{FUNDING}

The Chemicals in the Environment joint programme of the UK Department of Health and Department for Environment, Food and Rural Affairs.

\section{COMPETING INTERESTS}

None.

\section{Required licence statement}

The Corresponding Author has the right to grant on behalf of all authors and does grant on behalf of all authors, an exclusive licence (or non-exclusive for government employees) on a worldwide basis to the BMJ Publishing Group Ltd and its Licensees to permit this article (if accepted) to be published in Occupational and Environmental Medicine and any other BMJPGL products to exploit all subsidiary rights, as set out in our licence (http://oem.bmj.com/ifora/licence.pdf). 


\section{REFERENCES}

(1) WHO. Meta-analysis of time-series studies and panel studies of particulate matter and ozone. EUR/04/5042688 Bonn: World Health Organisation, 2004: 1-74.

(2) Schwarze PE, Ovrevik J, Lag M et al. Particulate matter properties and health effects: consistency of epidemiological and toxicological studies. Human \& Experimental Toxicology 2006; 25:559-579.

(3) Costa DL, Dreher KL. Bioavailable transition metals in particulate matter mediate cardiopulmonary injury in healthy and compromised animal models. Environ Health Perspect 1997; 105:1053-1060.

(4) Schaumann F, Borm PJA, Herbrich A et al. Metal-rich ambient particles (Particulate Matter(2.5)) cause airway inflammation in healthy subjects. Am J Resp and Crit Care Med 2004; 170:898-903.

(5) Merolla L, Richards RJ. In vitro effects of water-soluble metals present in UK particulate matter. Experimental Lung Research 2005; 31:671-683.

(6) Tao F, Gonzalez-Flecha B, Kobzik L. Reactive oxygen species in pulmonary inflammation by ambient particulates. Free Radical Biology and Medicine 2003; 35:327-340.

(7) Heal MR, Hibbs LR, Agius RM et al. Interpretation of variations in fine, coarse and black smoke particulate matter concentrations in a Northern European city. Atmos Environ 2005; 39:3711-3718.

(8) Heal MR, Hibbs LR, Agius RM et al. Total and water soluble trace metal content of urban background PM10, PM2.5 and Black Smoke in Edinburgh, UK. Atmos Environ 2005; 39:1417-1430.

(9) Prescott GJ, Cohen GR, Elton RA et al. Urban air pollution and cardiopulmonary ill health: a 14.5 year time series study. Occup Environ Med 1998; 55:697-704.

(10) Roemer W, Hoek G, Brunekreef B et al. PM10 elemental composition and acute respiratory health effects in European children (PEACE project). European Respiratory Journal 2000; 15:553-559.

(11) Tsai FC, Apte MG, Daisey JM. An exploratory analysis of the relationship between mortality and the chemical composition of airborne particulate matter. Inhal Toxicol 2000; 12:121-135.

(12) Sarnat JA, Marmur A, Klein M et al. Fine particle sources and cardiorespiratory morbidity: An application of chemical mass balance and factor analytical sourceapportionment methods. Environ Health Perspect 2008; 116:459-466.

(13) Lightbody JH, Hutchison G, Donaldson K et al. The influence of particle composition and size on in vitro and in vivo biological models (EPG1/3/147). Final Report to Defra. London 2003: 1-82. 